Hardy-Ramanujan Journal

Vol.27 (2004) 8-27

\title{
MEAN SQUARE OF THE HURWITZ ZETA-FUNCTION AND OTHER REMARKS
}

\author{
BY \\ R.BALASUBRAMANIAN AND K.RAMACHANDRA
}

(Accepted on 26-04-2005)

ABSTRACT. The Hurwitz zeta-function associated with the parameter $a(0<a \leq 1)$ is a generalisation of the Riemann zeta-function namely the case $a=1$. It is defined by

$$
\zeta(s, a)=\sum_{n=0}^{\infty}(n+a)^{-s},(s=\sigma+i t, \sigma>1)
$$

and its analytic continuation. In fact

$$
\zeta(s, a)=\sum_{n=0}^{\infty}\left((n+a)^{-s}-\int_{n}^{n+1} \frac{d u}{(u+a)^{s}}\right)+\frac{a^{1-s}}{s-1}
$$

gives the analytic continuation to $(\sigma>0)$. (This remark is due to E.LANDAU (see [EL]). A repetition of this process several times shows that

$$
\zeta(s, a)-\frac{a^{1-s}}{s-1}
$$

can be continued as an entire function to the whole plane. In $\operatorname{Re}(s) \geq-1, t \geq 2, \zeta(s, a)-$ $a^{-s}=O\left(t^{3}\right)$ and by the functional equation (see $\S 2$ ) it is

$$
O\left(\left(\frac{|s|}{2 \pi}\right)^{\frac{1}{2}-R e(s)}\right)
$$

in $\operatorname{Re}(s) \leq-1, t \geq 2$. From these facts we can deduce an 'Approximate functional equation' (see $\S 3$ ), which is a generalisation of the approximate functional equation for $\zeta(s)$. (The two are not very much different). Combining this with an important theorem due to van-derCORPUT, (see [ECT], Theorem 5.9), we prove

$$
T^{-\frac{1}{3}} \int_{T}^{T+T^{\frac{1}{3}}}\left|\zeta\left(\frac{1}{2}+i t, a\right)-a^{-\frac{1}{2}-i t}\right|^{2} d t \ll(\log T)^{3}
$$


uniformly in $a(0<a \leq 1)$. From this we deduce similar results for quasi- L functions (see $\left.[\mathrm{RB}, \mathrm{KR}]_{1}\right)$ and more general functions: Let $a_{1}, a_{2}, \ldots$ be any periodic sequence of complex numbers for which the sum over a period is zero. Let $b_{1}, b_{2}, \ldots$ be any sequence of complex numbers for which $\sum_{j=2}^{n}\left|b_{j}-b_{j-1}\right|+\left|b_{n}\right| \leq n^{\varepsilon}$ for every $\varepsilon>0$ and every $n \geq n_{0}(\varepsilon)$. Then we prove

$$
T^{-\frac{1}{3}} \int_{T}^{T+T^{\frac{1}{3}}}\left|\sum_{n=1}^{\infty} \frac{a_{n} b_{n}}{(n+a)^{\frac{1}{2}+i t}}\right|^{2} d t \leq T^{\varepsilon}
$$

for every $\varepsilon>0$ and every $T \geq T_{0}(\varepsilon)$ Here as usual $0<a \leq 1$ and $T_{0}(\varepsilon)$ is independent of $a$.

$\S 1$ INTRODUCTORY REMARKS. These concern problems on $\zeta(s, a)$ which need serious attention. We begin with M.N.HUXLEY'S recent important achievement [MNH]

$$
\zeta\left(\frac{1}{2}+i t\right)=O_{\varepsilon}\left(t^{\alpha+\varepsilon}\right), \alpha=\frac{32}{205}, t \geq 2 .
$$

The earlier important record was also due to him and it was $\alpha=\frac{89}{570}$. We wish him every success in his venture regarding improvement of (A.5) with $\frac{1}{3}$ replaced by $\frac{64}{205}$. The result

$$
\frac{1}{2 \pi} \int_{0}^{T}\left|\zeta\left(\frac{1}{2}+i t\right)\right|^{2} d t=\frac{T}{2 \pi} \log \frac{T}{2 \pi}+(2 \gamma-1) \frac{T}{2 \pi}+O\left(T^{\frac{1}{3}}\right)
$$

is due to R.BALASUBRAMANIAN [RB]. (Here as usual $\gamma=\sum_{n=1}^{\infty}\left(\frac{1}{n}-\int_{n}^{n+1} \frac{d u}{u}\right)$ is the EULER constant). It remains to consider

$$
\int_{0}^{T}\left|\sum_{n=1}^{\infty} \frac{a_{n} b_{n}}{(n+a)^{\frac{1}{2}+i t}}\right|^{2} d t
$$

and obtain an analogue of (1.2). Some work in the direction of extending (1.2) to $\zeta\left(\frac{1}{2}+\right.$ $i t, a)-a^{-\frac{1}{2}-i t}$ is in progress and it is due to a student of M.JUTILA. This extension gives (A.4) as a corollary and (A.4) implies

$$
\zeta\left(\frac{1}{2}+i t, a\right)-a^{-\frac{1}{2}-i t}=O\left(t^{\frac{1}{6}}(\log t)^{3}\right) .
$$

The result

$$
T^{-\frac{2}{3}} \int_{T}^{T+T^{\frac{2}{3}}}\left|\zeta\left(\frac{1}{2}+i t, a\right)-a^{-\frac{2}{2}-i t}\right|^{4} d t \ll_{\varepsilon} T^{\varepsilon}
$$

uniformly in $a$ seems to be hopeless at present although N.ZAVOROTNYI [NZ] has proved

$$
\int_{0}^{T}\left|\zeta\left(\frac{1}{2}+i t\right)\right|^{4} d t=T P(\log T)+O\left(T^{\frac{2}{3}+\varepsilon}\right) .
$$


where $P(x)$ is a certain polynomial of degree 4 in $x$. (For further work by Y.MOTOHASHI and A.IVIC see $[\mathrm{AI}]$ ). Even (1.4) with 1 in place of $\frac{2}{3}$ also seems to be hopeless. Mean value results such as (A.4) imply (by convexity principles)

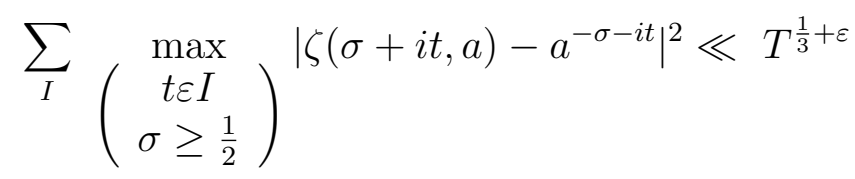

where I runs over all (abutting) unit intervals into which $\left[T, T+T^{\frac{1}{3}}\right]$ can be divided. (Similar remarks apply to all the mean value upper bounds over short intervals considered in this paper). It gives, as a corollary, the theorem

$$
\left|\zeta\left(\frac{1}{2}+i t, a\right)-a^{-\frac{1}{2}-i t}\right|<t^{\frac{1}{6}+\varepsilon}
$$

for all $t \geq t_{0}(\varepsilon)$. (This can certainly be obtained by the WEYL-HARDY-LITTLEWOOD method (see [ECT] Theorem 5.3)). The most important result in the theory of the Riemann zeta-function is, undoubtedly, the result

$$
|\zeta(\sigma+i t)| \leq C_{1} t^{(1-\sigma) 3 / 2} C_{2}(\log t)^{\frac{2}{3}},\left(\frac{1}{2} \leq \sigma \leq 1, t \geq 3\right)
$$

where $C_{1}>0$ and $C_{2}>0$ are absolute constants. This result is due to the Soviet Mathematician I.M.VINOGRADOV. (See the chapter on VINOGRADOFF's (both the names are the same) method in $[\mathrm{ECT}])$. He proved (1.6) with $(\log t)^{2 / 3}$ replaced by a higher power of $\log t$. Definitely he knew (1.6) and published

$$
\zeta(1+i t)=O\left((\log t)^{\frac{2}{3}}\right)
$$

also. Both (1.6) and (1.7) are nearly 50 years old. It is a great challenge to improve even (1.7). It is well-known that quasi Riemann hypothesis $(\zeta(s) \neq 0$ in $\sigma \geq 1-\delta$ for some constant $\delta>0$ ) implies easily

$$
\zeta(1+i t)=O(\log \log t), t \geq 100 .
$$

It is not hard to prove (1.6) for $\zeta(\sigma+i t, a)-a^{-\sigma-i t}$, (VINOGRADOFF's work gives (1.6) for this function as well). For the latest economical constants $C_{1}=76.2$ and $C_{2}=4.45$ in (1.6) see KEVIN FORD $[\mathrm{KF}]$. See also $[\mathrm{KR}, \mathrm{AS}]_{1}$.

$\S 2$ FUNCTIONAL EQUATION. (We borrow the functional equation for $\zeta(s, a)$ from $\S 2.17$ of $[\mathrm{ECT}])$. We have

$$
\zeta(s, a)=\sum_{n=0}^{\infty}(n+a)^{-s},(\sigma>1),
$$




$$
=\sum_{n=0}^{\infty}\left((n+a)^{-s}-\int_{n}^{n+1} \frac{d u}{(u+a)^{s}}\right)+\frac{a^{1-s}}{s-1},(\sigma>0),
$$

and repetition of this process several times shows that $\zeta(s, a)-\frac{a^{1-s}}{s-1}$ is entire. Also in $\sigma \geq-1, t \geq 2$ we have $\zeta(s, a)-a^{-s}=O\left(t^{3}\right)$. Again (see[ECT]) we have the functional equation

$$
\begin{aligned}
\zeta(s, a) & =\frac{2 \Gamma(1-s)}{(2 \pi)^{1-s}}\left\{\sin \left(\frac{s \pi}{2}\right) \sum_{m=1}^{\infty} \frac{\cos (2 \pi m a)}{m^{1-s}}\right. \\
& \left.+\cos \left(\frac{s \pi}{2}\right) \sum_{m=1}^{\infty} \frac{\sin (2 \pi m a)}{m^{1-s}}\right\},(\sigma<0)
\end{aligned}
$$

Note that $\left|\tan \left(\frac{s \pi}{2}\right)\right|$ lies between two positive constants for all $\sigma$ provided $t \geq 2$, and so the conversion factors

$$
\psi_{1}(s)=\frac{2 \Gamma(1-s)}{(2 \pi)^{1-s}} \sin \left(\frac{s \pi}{2}\right) \text { and } \psi_{2}(\mathrm{~s})=\frac{2 \Gamma(1-\mathrm{s})}{(2 \pi)^{1-\mathrm{s}}} \cos \left(\frac{\mathrm{s} \pi}{2}\right)
$$

are nearly the same (as that for the Riemann zeta-function) so far as the magnitudes are concerned. It is useful to record here that for $t \geq 2$, we have

$$
\left|\psi_{1}(s)\right|+\left|\psi_{2}(s)\right| \ll\left(\frac{|s|+1}{2 \pi}\right)^{\frac{1}{2}-\sigma}
$$

where the implied constant is absolute.

\section{§3. APPROXIMATE FUNCTIONAL EQUATION}

Let $0<\varepsilon \leq \frac{1}{100}, T \geq T_{0}(\varepsilon), L=\log T, \tau=L^{4}, h=\frac{10}{\varepsilon} L, 1 \leq X \leq T$,

$$
1 \leq Y \leq T, 1 \leq H \leq T^{\frac{1}{2}} \text { and } \mathrm{XY} \geq \frac{\mathrm{T}}{2 \pi}(1+\varepsilon) .
$$

Let $w=u+i v, s=\frac{1}{2}+i t, T \leq t \leq T+H, \zeta(s, a)=\sum_{n=0}^{\infty}(n+a)^{-s},(0<a \leq 1), \sigma>1$. (We put $s=\frac{1}{2}+i t$ for trivial simplification. The results are true with trivial modifications with $s=\sigma+i t$ for any $\sigma$ uniformly in any fixed interval $a_{0} \leq \sigma \leq b_{0}$ ). Then we have

$$
\begin{aligned}
& \sum_{n=1}^{\infty}(n+a)^{-s} \operatorname{Exp}\left(-\left(\frac{n+a}{x}\right)^{h}\right) \\
& =\frac{1}{2 \pi i} \int_{u=2,|v| \leq \tau}\left(\zeta(s+w, a)-a^{-s-w}\right) \Gamma\left(\frac{w}{h}+1\right) X^{w} \frac{d w}{w}+O\left(T^{-1}\right)
\end{aligned}
$$

Here we move the line of integration to $u=-\frac{5 L}{\varepsilon}$. (We use $\left|\Gamma\left(\frac{w}{h}+1\right)\right| \ll \exp \left(-\frac{-|\operatorname{Im}(w)|}{h}\right)$ in $\left.|R e(w)| \leq \frac{h}{2}\right)$. The pole at $w=0$ contributes $\zeta(s, a)-a^{-s}$ and the horizontal portions of 
the contour contribute $O\left(T^{-1}\right)$. We then use the functional equation and the fact that for $|\operatorname{Im}(z)| \geq 2$, we have

$$
\left|\psi_{1}(z)\right|+\left|\psi_{2}(z)\right| \ll\left(\frac{|z|+1}{2 \pi}\right)^{\frac{1}{2}-\operatorname{Re}(z)}
$$

a result mentioned already. We next break off the portion

$$
\sum_{m \geq Y} m^{s+w-1} \cos (2 \pi m a)
$$

of the series

$$
\sum_{m=1}^{\infty} m^{s+w-1} \cos (2 \pi m a)
$$

and estimate this tail portion by $O\left(Y^{\left(\frac{1-h}{2}\right)}\right)$, the total contribution from this being

$$
\begin{aligned}
& O\left(Y^{\left(\frac{1-h}{2}\right)} X^{-\frac{h}{2}}\left(\frac{T+H+\tau}{2 \pi}\right)^{\frac{1}{2}+\frac{h}{2}}\right) \\
& =O\left(\left\{\left(\frac{T+H+\tau}{2 \pi}\right)^{\frac{h}{2}}\left(\frac{1}{X Y}\right)^{\frac{h}{2}}(Y(T+H+\tau))^{\frac{1}{2}}\right\}\right) \\
& =O\left(\left\{\frac{T+H+\tau}{2 \pi} \frac{2 \pi}{T}(1+\varepsilon)^{-1}\right\}^{\frac{h}{2}}(Y(2 T))^{\frac{1}{2}}\right) \\
& =O\left(T(1+\varepsilon)^{-\frac{h}{2}} \log L\right) \\
& =O\left(T \operatorname{Exp}\left(-\left(\log (1+\varepsilon) \frac{5 L}{\varepsilon}\right)\right)=O\left(T^{-1}\right) .\right.
\end{aligned}
$$

We do a similar thing for $\left.\sum_{m=1}^{\infty} m^{s+w-1} \sin (2 \pi m a)\right)$. Thus we have

$$
\begin{aligned}
& \sum_{n=1}^{\infty}(n+a)^{-s} \operatorname{Exp}\left(-\left(\frac{n+a}{X}\right)^{h}\right) \\
& =\zeta(s, a)-a^{-s}+\frac{1}{2 \pi i} \int_{u=-5 L,|v| \leq \tau}\left(\psi_{1}(s+w) \sum_{m \leq Y} \frac{\cos (2 \pi m a)}{m^{1-s-w}}\right) X^{w} \Gamma\left(1+\frac{w}{h}\right) \frac{d w}{w} \\
& +\frac{1}{2 \pi i} \int_{u=-s L,|v| \leq \tau}\left(\psi_{2}(s+w) \sum \frac{\sin (2 \pi m a)}{m^{1-s-w}}\right) X^{w} \Gamma\left(1+\frac{w}{h}\right) \frac{d w}{w}+O\left(T^{-1}\right) \\
& =\zeta(s, a)-a^{-s}-\psi_{1}(s) \sum_{m \leq Y} \frac{\cos 2 \pi m a}{m^{1-s}} \\
& +\frac{1}{2 \pi i} \int_{u=\frac{1}{4},|v| \leq \tau}\left(\psi_{1}(s+w) \sum_{m \leq Y} \frac{\cos (2 \pi m a)}{m^{1-s-w}}\right) X^{w} \Gamma\left(1+\frac{w}{h}\right) \frac{d w}{w}
\end{aligned}
$$




$$
\begin{aligned}
& -\psi_{2}(s) \sum_{m \leq Y} \frac{\sin (2 \pi m a)}{m^{1-s}}+\frac{1}{2 \pi i} \int_{a=\frac{1}{4},|v| \leq \tau}\left(\psi_{2}(s+w) \sum_{m \leq Y} \frac{\sin (2 \pi m a)}{m^{1-s-w}}\right) X^{w} \Gamma\left(1+\frac{w}{h}\right) \frac{d w}{w} \\
& +O\left(T^{-1}\right) .
\end{aligned}
$$

Further if we impose

$$
\frac{T+H}{2 \pi}(1+3 \varepsilon) \geq X Y
$$

we can throw away

$$
\frac{1}{2 \pi i} \int_{u=\frac{1}{4}|v| \leq \tau}\left(\psi_{1}(s+w) \sum_{m \leq(1-9 \varepsilon) Y} \frac{\cos (2 \pi m a)}{m^{1-s-w}}\right) X^{W} \Gamma\left(1+\frac{w}{h}\right) \frac{d w}{w}
$$

since it is (on moving the line of integration $t_{0} u=\frac{5 L}{\epsilon}$ )

$$
\begin{aligned}
& O\left(X^{\frac{h}{2}}((1-9 \varepsilon) Y)^{\frac{1}{2}+\frac{h}{2}}\left(\frac{T}{2 \pi}\right)^{-\left(\frac{h+1}{2}\right)}\right) \\
& =O\left(\left(X Y(1-9 \varepsilon)^{\frac{h}{2}} Y^{\frac{1}{2}}\left(\frac{T}{2 \pi}\right)^{-\frac{h}{2}}\right)\right. \\
& =O\left(((1-9 \varepsilon)(1+4 \varepsilon))^{\frac{h}{2}} T\right)-O\left(T^{-1}\right) .
\end{aligned}
$$

Collecting we have

THEOREM 3.1 Let $0<\varepsilon \leq \frac{1}{100}, T \geq T_{0}(\varepsilon), s=\frac{1}{2}+i t, T \leq t \leq T+H, L=\log T, \tau=$ $L^{4}, h=\frac{10}{\varepsilon} L, 1 \leq X \leq T, 1 \leq Y \leq T, X Y \geq \frac{T}{2 \pi}(1+\varepsilon), w=u+i v$, and let $\zeta(s, a)$ be as before.

Then, we have,

$$
\begin{aligned}
& \zeta(s, a)-a^{-s}=\sum_{n=1}^{\infty}(n+a)^{-s} \operatorname{Exp}\left(-\left(\frac{n+a}{X}\right)^{h}\right) \\
& +\psi_{1}(s) \sum_{m \leq Y} \frac{\cos (2 \pi m a)}{m^{1-s}}+\psi_{2}(s) \sum_{m \leq Y} \frac{\sin (2 \pi m a)}{m^{1-s}} \\
& -\frac{1}{2 \pi i} \int_{u=\frac{1}{4},|v| \leq \tau}\left(\psi_{1}(s+w) \sum_{m \leq Y} \frac{\cos (2 \pi m a)}{m^{1-s-w}}\right) X^{w}+\Gamma\left(1+\frac{w}{h}\right) \frac{d w}{w} \\
& -\frac{1}{2 \pi i} \int_{u=\frac{1}{4}|v| \leq \tau}\left(\psi_{2}(s+w) \sum_{m \leq Y} \frac{\sin (2 \pi m a)}{m^{1-s-w}}\right) X^{w} \Gamma\left(1+\frac{w}{h}\right) \frac{d w}{w} \\
& +O\left(T^{-1}\right)
\end{aligned}
$$

Here, we have, for $|\operatorname{Im}(z)| \geq 1$, as noted already in (3.3)

$$
\left|\psi_{1}(z)\right|+\left|\psi_{2}(z)\right| \ll\left(\frac{|z|+1}{2 \pi}\right)^{\frac{1}{2}-\operatorname{Re}(z)} .
$$


Also in the last two integrals we can throw away

$$
\sum_{m \leq(1-9 \varepsilon) Y} \frac{\cos (2 \pi m a)}{m^{1-s-w}} \text { and } \sum_{\mathrm{m} \leq(1-9 \varepsilon) \mathrm{Y}} \frac{\sin (2 \pi m a)}{m^{1-\mathrm{s}-\mathrm{w}}}
$$

and retain only $\sum_{(1-9 \varepsilon) Y \leq m \leq Y} \ldots$, provided we impose an additional condition

$$
\frac{T+H}{2 \pi}(1+3 \varepsilon) \geq X Y \text {. }
$$

$\S 4$. PROOF OF (A.4). The proof is very much similar to that of Lemmas 1 to 9 (pages 357-359 putting $q=1$ ) in $[\mathrm{RB}, \mathrm{KR}]_{2}$. A key lemma is the following theorem due to van-derCORPUT (see theorem 5.9 of [ECT]).

LEMMA 4.1 If $f(x)$ is real and twice differentiable and $0<\lambda_{2} \leq f^{\prime \prime}(x) \leq h \lambda_{2}$ (or $0<\lambda_{2} \leq$ $\left.-f^{\prime \prime}(x) \leq h \lambda_{2}\right)$ through out the interval $[a, b]$ and $b \geq a+1$, then

$$
\sum_{a \leq n \leq b} \exp (2 \pi i f(n))=O\left(h(b-a) \lambda_{2}^{\frac{1}{2}}\right)+O\left(\lambda_{2}^{-\frac{1}{2}}\right) .
$$

REMARK. We have quoted theorem 5.9 of [ECT] in the notation employed there. The $h$ of this lemma (and $a$ ) should not be confused with $h$ (and $a$ ) of the present paper.

There are five expressions (apart from $O\left(T^{-1}\right)$ ) on the RHS of (3.5). Denote by $F(s)$ any of these. It suffices to prove (with $H=T^{\frac{1}{3}}$ ) the inequality

$$
\frac{1}{H} \int_{T}^{T+H}|F(s)|^{2} d t \ll(\log T)^{2} .
$$

We do this for

$$
F(s)=\psi_{1}(s) \sum_{m \leq Y} \frac{\cos (2 \pi m a)}{m^{1-s}},\left(s=\frac{1}{2}+i t\right) .
$$

(Note that $\left.\left|\psi_{1}\left(\frac{1}{2}+i t\right)\right|=\left|\psi_{2}\left(\frac{1}{2}+i t\right)\right| \ll 1\right)$. The rest of the terms can be handled similarly.

LEMMA 4.2. Let $X=Y=T^{\frac{1}{2}}, s=\frac{1}{2}+i t$ and

$$
S_{1} \equiv S_{1}(t) \equiv \sum_{1 \leq m \leq Y} \frac{\cos (2 \pi m a)}{m^{-s}}
$$

Let $H \geq C \log T$, where $C>0$ is a large constant and $0 \leq u_{i} \leq H /(4 \alpha)(i=$ $1,2,3, \ldots, \alpha)$, where $\alpha$ is a natural number at our choice. We put $\alpha=[100 \log T]$ and we have

$$
\int_{T}^{T+H}\left|S_{1}\right|^{2} d t \leq\left(\frac{H}{4 \alpha}\right)^{-\alpha} \int_{0}^{\frac{H}{(4 \alpha)}} \ldots \int_{0}^{\frac{H}{(4 \alpha)}}\left(\int_{T-V}^{T+H+V}\left|S_{1}(t)\right|^{2} d t\right) d u_{1} \ldots d u_{\alpha}
$$


where $V=u_{1}+u_{2}+\ldots+u_{\alpha}$ and the integration with respect to $u_{1}, \ldots, u_{\alpha}$ is over the $\alpha-$ dimensional cube defined just now.

REMARK. This idea has been used extensively by R.BALASUBRAMANIAN[RB]. The proof is simple and is left as an exercise to the reader.

We split up the range $[1, \mathrm{Y}]$ into $m=1,[2,4),[4,8), \ldots$

LEMMA 4.3 The right hand side of (4.5) is

$$
\leq 4 \log T\left(\frac{H}{4 \alpha}\right)^{-\alpha} \sum_{U} \int_{0}^{\frac{H}{4 \alpha}} \ldots \int_{0}^{\frac{H}{4 \alpha}}\left(\int_{T-V}^{T+H+V}\left|S_{2}(t)\right|^{2} d t\right) d u_{1} \ldots d u_{\alpha}
$$

where

$$
S_{2} \equiv S_{2}(t)=\sum_{U \leq m \leq U^{\prime}}(\cos (2 \pi m a)) m^{s-1}
$$

$U, U^{\prime}$ being the initial and final points of the intervals mentioned. The last of these intervals may be a portion of an interval $\left[2^{k}, 2^{k+1}\right)$. Trivially the contribution from $m=1$ is $O(H \log T)$.

PROOF. Follows from $\left|S_{1}\right|^{2}=\left|\sum_{U} S_{2}\right|^{2} \leq 4 \log T \sum_{U}\left|S_{2}\right|^{2}$

Let

$$
f\left(m_{1}, m_{2}\right)=\cos \left(2 \pi m_{1} a\right) \cos \left(2 \pi m_{2} a\right)\left(\frac{m_{1}}{m_{2}}\right)^{i t}\left(m_{1} m_{2}\right)^{-\frac{1}{2}}
$$

and let $D$ denote the condition defined by

$$
D \equiv\left\{U \leq m_{1} \leq U^{\prime}, U \leq m_{2} \leq U^{\prime}\right\}
$$

Then

$$
\left|S_{2}\right|^{2}=\sum_{\left(m_{1}, m_{2}\right) \epsilon D} f\left(m_{1}, m_{2}\right)
$$

and

$$
\begin{gathered}
\int_{T-V}^{T+H+V}\left|S_{2}(t)\right|^{2} \\
=\sum_{m_{1}, m_{2} \epsilon D} \cos \left(2 \pi m_{1} a\right) \cos \left(2 \pi m_{2} a\right)\left(m_{1} m_{2}\right)^{-\frac{1}{2}} \int_{T-V}^{T+H+V}\left(\frac{m_{1}}{m_{2}}\right)^{i t} d t .
\end{gathered}
$$

Here the terms with $m_{1}=m_{2}$ contribute

$$
O\left(H \sum_{U \leq m \leq U^{\prime}}(\cos (2 \pi m a))^{2} m^{-1}\right)=O(H) .
$$


Consider contributions from non-diagonal terms. Since

$$
\left|\log \frac{m_{1}}{m_{2}}\right| \geq\left|\frac{m_{1}-m_{2}}{m_{1}+m_{2}}\right|
$$

the contribution is

$$
\sum_{m_{1} \neq m_{2}}\left(m_{1} m_{2}\right)^{-\frac{1}{2}}\left|\frac{m_{1}+m_{2}}{m_{1}-m_{2}}\right|^{\alpha}
$$

We dispose of those terms with $\left|m_{1}-m_{2}\right| \geq \Delta$ (a parameter at our choice). These contribute an amount not more than

$$
\left(\frac{60 \alpha U}{H \Delta}\right)^{\alpha} \sum\left(m_{1} m_{2}\right)^{-\frac{1}{2}} \ll T^{-1}
$$

provided $\Delta=100 \alpha U H^{-1}$. (As stated before we impose on $H$, the condition $H \geq C \log T$, where $C>0$ is a large constant). Here the sum of all such terms from all $U$ is $O(1)$. Hence it suffices to consider those coming from $D$ with

$$
\left|m_{1}-m_{2}\right| \leq 100 \alpha U H^{-1}
$$

(Note that the condition $H \geq C \log T$ ensures that the RHS of (4.8) is $\leq 10^{-4} C^{-1} U$ and so $\leq \varepsilon U$ for all $\varepsilon(0<\varepsilon<1)$ if $C$ is large enough depending on $\varepsilon)$. Of course it suffices to estimate contributions from those terms $\left(m_{1}, m_{2}\right)$ with $m_{1}>m_{2}$. (The terms with $m_{1}<m_{2}$ can be treated similarly). We have to estimate

$$
-i \sum_{m_{1}>m_{2}}\left(\left(\frac{m_{1}}{m_{2}}\right)^{i(T+H+V)}-\left(\frac{m_{1}}{m_{2}}\right)^{i(T-V)}\right)\left(m_{1}, m_{2}\right)^{-\frac{1}{2}}\left(\log \frac{m_{1}}{m_{2}}\right)^{-1} g\left(m_{1}, m_{2}\right)
$$

where $g\left(m_{1}, m_{2}\right)=\cos \left(2 \pi m_{1} a\right) \cos \left(2 \pi m_{2} a\right)$.

In place of $g\left(m_{1}, m_{2}\right)$ we can consider $\operatorname{Exp}\left(2 \pi a\left( \pm m_{1} \pm m_{2}\right) i\right)$ for a fixed combination of signs \pm . We now write $m_{1}=m_{2}+r$. Thus it suffices to estimate

$$
4 \sum_{r} \sum_{m_{2}} \frac{\operatorname{Exp}\left(i t \log \left(1+\frac{r}{m_{2}}\right)\right) \operatorname{Exp}\left(2 \pi i a\left( \pm m_{2} \pm m_{2} \pm r\right)\right)}{\left(m_{2}\left(m_{2}+r\right)\right)^{\frac{1}{2}} \log \left(1+\frac{r}{m_{2}}\right)}
$$

where the conditions of summation are $U \leq m_{2} \leq U^{\prime}$ and $1 \leq r \leq 100 \alpha U H^{-1}$ and $t$ denotes a number lying between $\frac{T}{2}$ and $3 T$. Because of the summation conditions on $r$, it is not hard to verify that for each fixed $r$

$$
\left(m_{2}\left(m_{2}+r\right)\right)^{\frac{1}{2}} \log \left(1+\frac{r}{m_{2}}\right)
$$


is monotonic in $m_{2}$ and lies between two constant multiples of $r$. (We assume $100 \alpha U H^{-1} \geq 1$. Other wise there is no $r$ ). To estimate the innermost sum in (4.10) we use Lemma 1, which is very important. We take

$$
\left.\begin{array}{c}
f(x)=\left\{\frac{t}{2 \pi} \log \left(1+\frac{r}{x}\right)+a( \pm x \pm x \pm r),\right. \\
a=U \text { and } \mathrm{b}=\mathrm{U}^{\prime} .
\end{array}\right\} .
$$

We see that $f^{\prime \prime}(x)$ lies between two positive constant multiples of $\operatorname{Tr} x^{-3}$ and so the inner sum in (4.10) is $r^{-1}$ times a quantity which is

$$
\ll U\left(\frac{T r}{U^{3}}\right)^{\frac{1}{2}}+\left(\frac{U^{3}}{T r}\right)^{\frac{1}{2}} .
$$

Hence the sum (4.10) is majorised by

$$
\begin{aligned}
& \sum_{r \leq 100 \alpha U H^{-1}}\left\{\frac{1}{r}\left(\frac{T r}{U}\right)^{\frac{1}{2}}+\left(\frac{U^{3}}{T r}\right)^{\frac{1}{2}}\right\} \\
& \ll\left(\frac{\alpha T}{H}\right)^{\frac{1}{2}}+\left(\frac{U^{3}}{T}\right)^{\frac{1}{2}} .
\end{aligned}
$$

Summation over $U\left(\leq T^{\frac{1}{2}}\right.$ gives a factor $\log T$ for the first term and $O\left(T^{\frac{1}{4}}\right)$ for the second. Collecting we have

LEMMA 4.4. Let $S_{1}(t) \equiv S_{1} \equiv \sum_{m \leq Y} m^{s-1} \cos (2 \pi m a), T \geq H \geq C \log T$ where $C>0$ is a large constant. Then

$$
\begin{aligned}
& \int_{T}^{T+H}\left|S_{1}(t)\right|^{2} d t \\
& \ll H \log T+T^{\frac{1}{4}}(\log T)^{2}+\left(\frac{T}{H}\right)^{\frac{1}{2}}(\log T)^{\frac{5}{2}} .
\end{aligned}
$$

REMARK. Taking $H=T^{\frac{1}{3}}$ we see that the expression $(4.15)$ is $O\left(H(\log T)^{\frac{5}{2}}\right)$. The two integrals in (3.5) can be treated by first estimating the sum $\sum_{m \leq Y} \ldots$ and then noting that

$$
\int_{u=\frac{1}{4},|v| \leq \tau}\left|\Gamma\left(1+\frac{w}{h}\right)\right|\left|\frac{d w}{w}\right| \ll \log h \ll \log \log T
$$

This can be seen as follows. $\left|\Gamma\left(1+\frac{w}{h}\right)\right| \ll \operatorname{Exp}\left(-\left|\frac{\operatorname{Im}(w)}{h}\right|\right) \ll\left|\frac{h}{\operatorname{Im}(w)}\right|^{2}$ (if $\left.u=\frac{1}{4}\right)$, also $\left|\Gamma\left(1+\frac{w}{h}\right)\right| \ll 1$. Again $\left|\frac{1}{w}\right| \ll \min \left(1,|v|^{-1}\right)$ if $u=\frac{1}{4}$. Thus

$$
\int_{|v| \leq 1}\left|\Gamma\left(1+\frac{w}{h}\right)\right|\left|\frac{d w}{w}\right| \ll \int_{|v| \leq 1} d v \ll 1
$$


and

$$
\begin{gathered}
\int_{h \geq|v| \geq 1}\left|\Gamma\left(1+\frac{w}{h}\right)\right|\left|\frac{d v}{v}\right| \ll \int_{1}^{h}\left|\frac{d v}{v}\right| \ll 1 \\
\int_{\tau \geq|v| \geq 1}\left|\Gamma\left(1+\frac{w}{h}\right)\right|\left|\frac{d v}{v}\right| \ll \int_{h}^{\infty}\left(\frac{h}{v}\right)^{2} \frac{d v}{v} \ll 1 .
\end{gathered}
$$

Thus compromising a little we get $O\left((\log T)^{\frac{5}{2}} \log \log T\right)=O\left((\log T)^{3}\right)$ for the LHS of (A.4). We state this as

THEOREM 4.1. We have, for $H=T^{\frac{1}{3}}$,

$$
\frac{1}{H} \int_{T}^{T+H}\left|\zeta\left(\frac{1}{2}+i t, a\right)-a^{-\frac{1}{2}-i t}\right|^{2} d t \ll(\log T)^{3} .
$$

\section{§5. FURTHER APPLICATIONS OF THE APPROXIMATE FUNCTIONAL}

EQUATION. We assume that $a_{1}, a_{2}, \ldots$ is a periodic sequence of complex numbers (with period $k$ ) and that the sum over a period zero. We begin with the remark that if $\alpha$ is any number with $0<\alpha \leq 1$, (in this section we use $\alpha$ and not a) we have (for $\sigma>1$ )

$$
\begin{gathered}
F(s) \equiv \sum_{n=1}^{\infty} \frac{a_{n}}{(n+\alpha)^{s}}=\sum_{l=1}^{k} a_{l} \sum_{\substack{n=\infty \\
\nu=0}}^{\nu=\infty}(n+\alpha)^{-s} \\
=\sum_{l=1}^{k} a_{l} \sum_{\nu=0}^{\infty}(l+\nu k+\alpha)^{s}=\sum_{l=1}^{k} a_{l} k^{-s} \sum_{\nu=0}^{\infty}\left(\nu+\frac{l+\alpha}{k}\right)^{-s}
\end{gathered}
$$

and so (for $s=\frac{1}{2}+i t$ ),

$$
|F(s)|^{2} \leq 2 \sum_{l=1}^{k} A^{2}\left\{\left|\sum_{\nu=1}^{\infty}\left(\nu+\frac{l+\alpha}{k}\right)^{-s}\right|^{2}+\left(\left|\frac{k}{l+\alpha}\right|\right)\right\},
$$

where $A=\max \left|a_{l}\right|$ taken over $\mid \leq l \leq k$. Thus we have, (for $H=T^{\frac{1}{3}}$,

$$
\frac{1}{H} \int_{T}^{T+H}|F(s)|_{\sigma=\frac{1}{2}}^{2} d t \ll A^{2}(\log T)^{3}+A^{2} k \log k
$$

(uniformly in $\alpha$ ) by theorem 4.1. (in these calculations we mean the infinite sum to be the analytic continuation of the Hurwitz zeta-function). We state this as a part of the following Theorem.

THEOREM 5.1. Let $a_{1}, a_{2}, \ldots$ be any periodic sequence of complex numbers with period $k(\geq 1)$ and the sum over a period zero. 
Let $\max _{j}\left|a_{j}\right| \leq A$. Then with any $\alpha(0<\alpha \leq 1)$ and $H=T^{\frac{1}{3}}$ we have (for $T \geq 2$ )

$$
\frac{1}{H} \int_{T}^{T+H}\left|\sum_{n=1}^{\infty} \frac{a_{n}}{(n+\alpha)^{s}}\right|_{\sigma=\frac{1}{2}}^{2} d t \ll A^{2} k(\log T)^{3}+A^{2} k \log k
$$

uniformly in $\alpha$. Further let $b_{0}=0, b_{1}, b_{2}, \ldots$ be any sequence of complex numbers satisfying

$$
\sum_{j=1}^{N}\left|b_{j}-b_{j-1}\right|+\left|b_{N}\right| \ll N^{\varepsilon}
$$

for every $\varepsilon>0$ and every $N \geq 1$. Then with $H=T^{\frac{1}{3}}$, we have,

$$
\frac{1}{H} \int_{T}^{T+H}\left|\sum_{n=1}^{\infty} \frac{a_{n} b_{n}}{(n+\alpha)^{s}}\right|_{\sigma=\frac{1}{2}}^{2} d t \ll_{\varepsilon} T^{\varepsilon}
$$

for every $\varepsilon>0$ and all $T \geq 1$ uniformly in $\alpha$.

REMARKS. The result (5.1) is already proved. In (5.1) and (5.3) we can assume that $T \geq T_{0}(\varepsilon)$, since otherwise the LHS expressions are bounded. The rest of this section is devoted for the proof of (5.3).

We have

$$
\begin{aligned}
& \sum_{n=1}^{N} \frac{a_{n} b_{n}}{(n+\alpha)^{s}}=A_{1} b_{1}+\left(A_{2}-A_{1}\right) b_{2}+\left(A_{3}-A_{2}\right) b_{3}+\ldots \\
& \ldots+\left(A_{N}-A_{N-1}\right) b_{N} \\
& =A_{1}\left(b_{1}-b_{2}\right)+A_{2}\left(b_{2}-b_{3}\right)+\ldots+A_{N-1}\left(b_{N-1}-b_{N}\right)+A_{N} b_{N}
\end{aligned}
$$

where

$$
A_{j}=\sum_{n \leq j} \frac{a_{n}}{(n+\alpha)^{s}} .
$$

So

$$
\begin{aligned}
& \left|\sum_{n=1}^{N} \frac{a_{n} b_{n}}{(n+\alpha)^{s}}\right|^{2} \leq 2\left(\sum_{j \leq N-1}\left|A_{j}\right|^{2}\left|b_{j}-b_{j+1}\right|\right) \sum_{j \leq N}\left|b_{j}-b_{j+1}\right| \\
& +2\left|A_{N}\right|^{2}\left|b_{N}\right|^{2} \\
& \leq 2 \max _{j \leq N}\left(\left|A_{j}^{2}\right|+1\right)\left(\left(\sum_{j \leq N-1}\left|b_{j}-b_{j+1}\right|\right)^{2}+\left|b_{N}\right|^{2}\right)
\end{aligned}
$$




$$
\begin{aligned}
& \leq 2 \max _{j \leq N}\left(\left|A_{j}\right|^{2}+1\right)\left(\sum_{j \leq N-1}\left|b_{j}-b_{j+1}\right|+\left|b_{N}\right|\right)^{2} \\
& \leq 2\left(\max _{j \leq N}\left|A_{j}\right|^{2}+1\right) N^{\varepsilon}
\end{aligned}
$$

by hypothesis (5.2). Hence it remains to estimate

$$
\int_{T}^{T+H}\left|A_{j}\right|^{2} d t(j=1,2, \ldots) .
$$

(It is not hard to verify the analogue of (5.4) namely

$$
\left|\sum_{M+1}^{M+N} \frac{a_{n} b_{n}}{(n+\alpha)^{s}}\right| \ll_{\varepsilon} \frac{M^{\varepsilon}}{M^{\sigma}}
$$

provided $N \leq M$, and so the series

$$
\sum_{n=1}^{\infty} \frac{a_{n} b_{n}}{(n+\alpha)^{s}}
$$

is convergent for $\sigma \geq \delta$ uniformly in any compact subset of $\sigma \geq \delta$ ). More over

$$
\sum_{n \geq M} \frac{a_{n}}{(n+\alpha)^{s}}=O_{\varepsilon, \delta}\left(M^{-\sigma+\varepsilon}\right)
$$

for $M \geq T^{1+\varepsilon}(T \leq t \leq 2 T)$ uniformly for $\sigma \geq \delta$ (see $\left.[\mathrm{RB}, \mathrm{KR}]_{1}\right)$.

Although $\sum_{n=1}^{\infty} \frac{a_{n}}{(n+\alpha)^{s}}$ does not have an approximate functional equation we can split up (as noted before Theorem 5.1)

$$
F(s)=\sum_{n=1}^{\infty} \frac{a_{n}}{(n+\alpha)^{s}}=\sum_{l=1}^{k} a_{l} \sum_{\nu=0}^{\infty}\left(\nu+\frac{l+\alpha}{k}\right)^{-s}
$$

and the innermost sum is the Hurwitz zeta-function and it has an approximate functional equation. Thus we can attack, (we put $\sigma=\frac{1}{2}$ in the rest of this section),

$$
A_{j}(s)+O(1)=\sum_{n=1}^{j} \frac{a_{n}}{(n+\alpha)^{s}}=\sum_{l=1}^{k} a_{l} k^{-s} \sum_{\nu=0}^{\left[\frac{j}{k}\right]}\left(\nu+\frac{l+\alpha}{k}\right)^{-s}+O(1) .
$$

Here by $\nu=0$ to $[j / k]$, we mean the truncation at $\nu=\left[\frac{j}{k}\right]$ contribution of the series $\sum_{\nu=0}^{\infty}\left(\nu+\frac{l+\alpha}{k}\right)^{-s}$

Now

$$
\left|A_{j}(s)\right| \ll A k^{-\frac{1}{2}} \sum_{l=1}^{k}\left|\sum_{\nu=0}^{[j / k]}\left(\nu+\frac{l+\alpha}{k}\right)^{-s}\right|
$$


Hence it suffices to estimate

$$
\int_{T}^{T+H}\left|\sum_{\nu=0}^{[j / k]}\left(\nu+\frac{l+\alpha}{k}\right)^{-s}\right|^{2} d t
$$

where $j=1,2, \ldots,\left[T^{1+\varepsilon}\right]$ (see $\left.[\mathrm{RB}, \mathrm{KR}]_{1}\right)$. In fact we can stop at $j=[k T](T \leq t \leq T+H)$ see theorem 1.3.2 of $[\mathrm{KR}]_{1}$. (Here $\zeta(s)$ has been considered. But argument goes through for $\zeta(s, a)$ by the same method). We do not attempt uniformity in $A, k$ and etc. Hereafter we write

$$
B_{j}(s)=\sum_{\nu=0}^{j}\left(\nu+\frac{l+\alpha}{k}\right)^{-s}
$$

where $l, k$ and $\alpha$ are fixed $(0<\alpha \leq 1)$. We proceed to estimate

$$
\int_{T}^{T+H}\left|B_{j}(s)-a^{-s}\right|_{\sigma=\frac{1}{2}}^{2} d t \text { where } a=\frac{l+\alpha}{\mathrm{k}} .
$$

Here $1 \leq j \leq k T$. We can assume $j \geq 2$ since for $j=1(5.8)$ is $O(1)$. It is well known $\left(\right.$ see $\left.[\mathrm{KR}]_{2}\right)$ that

$$
\begin{aligned}
& \sum_{n=1}^{j}(n+a)^{-s}=\frac{1}{2 \pi i} \int_{2-i \infty}^{2+i \infty}\left(\zeta(s+w)-a^{-s-w}\right) \frac{\left(j+a+\frac{1}{2}\right)^{w}}{w} d w \\
& =\frac{1}{2 \pi i} \int_{C-i T, C=1-\sigma+(\log j)^{-1}}^{C+i T}\left(\zeta(s+w)-a^{-s-w}\right) \frac{\left(j+a+\frac{1}{2}\right)^{w}}{w} d w \\
& +O\left(\sum_{n=1}^{\infty}\left(\left(\frac{j+a+\frac{1}{2}}{n+a}\right)^{1-\sigma+(\log j)^{-1}} n^{-\sigma} T^{-1}\left|\log \frac{j+a+\frac{1}{2}}{n+a}\right|^{-1}\right) .\right.
\end{aligned}
$$

We split the sum inside O-term to $\sum_{1}=\sum_{j / 2 \leq n \leq 3 \frac{j}{2}}$,

$$
\sum_{2}=\sum_{n \leq j / 2} \text { and } \sum_{3}=\sum_{n \geq 3 \frac{j}{2}}
$$

We have (easily)

$$
\begin{aligned}
& \left|\sum_{1}\right| \ll \frac{j^{1-\sigma}}{T} \log j, \text { and } \\
& \left|\sum_{2}\right|+\left|\sum_{3}\right| \ll \sum_{n=1}^{\infty} \frac{1}{T}\left(j^{1-\sigma} n^{-1-\frac{1}{\log j}}\right)
\end{aligned}
$$

Hence with $(T \leq t \leq T+H)$, we have (with any $V, 10 T^{\frac{1}{3}} \geq V \geq \frac{1}{10} T^{\frac{1}{3}}$ )

$$
\sum_{1 \leq n \leq j}(n+a)^{-s}=\frac{1}{2 \pi i} \int_{C-i V}^{C+i V}\left(\zeta(s+w, a)-a^{-s-w}\right) \frac{\left(j+a+\frac{1}{2}\right)^{w}}{w} d w
$$




$$
+O\left(j^{1-\sigma}(\log j) V^{-1}\right),
$$

and so (note that it suffices to consider $\sum_{j \leq n \leq 2 j} \ldots$.)

$$
\begin{aligned}
& \sum_{j \leq n \leq 2 j}(n+a)^{-s} \\
= & \frac{1}{2 \pi i} \int_{C-i V}^{C+i V}\left(\zeta(s+w, a)-a^{-s-w}\right) \frac{\left(2 j+a+\frac{1}{2}\right)^{w}-\left(j+a+\frac{1}{2}\right)^{w}}{w} d w \\
+ & O\left(\frac{j^{1-\sigma} \log j}{V}\right) \\
= & \frac{1}{2 \pi i} \int_{-i V}^{i V}\left(\zeta(s+w, a)-a^{-s-w}\right) \frac{\left(2 j+a+\frac{1}{2}\right)^{w}-\left(j+a+\frac{1}{2}\right)^{w}}{w} d w \\
+ & O\left(j^{1-\sigma}(\log j) V^{-1}\right)+O\left(j^{1-\sigma} V^{-1}(\log T)^{100}\right) .
\end{aligned}
$$

In arriving at (5.10) we have used the following Lemma.

LEMMA 5.1 Let $H=T^{\frac{1}{3}}$ and $T \geq T_{0}$. Then

$$
\int_{\frac{1}{2}}^{1} \int_{T}^{T+H}\left|\zeta(\sigma+i t, a)-a^{-\sigma-i t}\right|^{2} d t d \sigma \leq H(\log T)^{20} .
$$

PROOF. The proof follows from theorem 4.1 by standard convexity arguments (given for completeness in $\S 6$ of the present paper). We remark that it is possible to sharpen (5.11), but we do not attempt it here. We would like to mention here that Lemma 3.4 of $[\mathrm{KR}, \mathrm{AS}]_{2}$ can be deduced from $\S 6$ of the present paper.

We are now in a position to complete the proof of (5.3). We have (by theorem 4.1, the mean-square of the integral in $(5.10))$ is $\left.\ll(\log T)^{10}\right)$ for $1 \leq j \leq k T$

$$
\frac{1}{H} \int_{T}^{T+H}\left|\sum_{j \leq n \leq 2 j}(n+a)^{-s}\right|_{\sigma=\frac{1}{2}}^{2} d t \ll(\log T)^{10}+j V^{-2}(\log T)^{100}
$$

where $H=T^{\frac{1}{3}}$ and $10 T^{\frac{1}{3}} \geq U \geq \frac{1}{10} T^{\frac{1}{3}}$. Thus we are through if $j \ll T^{\frac{2}{3}}$. Now if $j \geq T^{\frac{2}{3}-\varepsilon}$ approximate functional equation (Theorems 3.1 and 4.1) gives with $Y=T^{\frac{2}{3}-\varepsilon}$ (see (3.6)) the fact that with $X=T^{\frac{1}{3}+\epsilon}$ it suffices to prove that

$$
\frac{1}{H} \int_{T}^{T+H}\left|\sum_{n=1}^{\infty}(n+a)^{-s} \operatorname{Exp}\left(-\left(\frac{n+a}{X}\right)^{h}\right)\right|^{2} d t \ll T^{2 \varepsilon}
$$


and this follows by a theorem of H.L.MONTGOMERY and R.C.VAUGHAN (see[KR $]_{3}$ ).

Thus (5.3) is completely proved and thus theorem 5.1 is completely proved.

§6.PROOF OF LEMMA 5.1 The notation in this section is different and should not be confused with that in the earlier sections. Let $F(s)(s=\sigma+i t)$ be analytic in the rectangle

$$
R_{1}: a \leq \sigma \leq b, T \leq t \leq T+H
$$

where $a<b$ and $|F(s)|$ is bounded (above) there by $T^{C}$ (for some constant $C>0$ ). Consider the rectangle

$$
R_{2}: a+\delta \leq \sigma \leq b-\eta, T-\log T \leq t \leq T+H-\log T
$$

where $\delta$ and $\eta$ are smaller than $T^{-100}$ (or some such small or smaller quantities). Note that if $F^{\prime}(s)$ is not too big then $\int|F(s)| d t$ does not alter much over any two close vertical lines contained in $R_{1}$ and so $\int_{(\sigma)} \int_{(t)}|F(s)| d t$ is nearly the same as $\left.\left(\int_{(t), \sigma=\sigma_{0}}|F(s)| d t\right) \int_{(\sigma)} d \sigma\right)$, where $\sigma_{0}$ is any fixed $\sigma$.

Hence it suffices to consider upper bounds for

$$
\int_{a+\delta \leq \sigma_{0} \leq b-\eta} \int_{T-\log T}^{T+H-\log T}|F(s)| d t
$$

in terms of

$$
M_{1} \equiv \int_{T, \sigma=a}^{T+H}|F(s)| d t \text { or } \mathrm{M}_{2} \equiv \int_{\mathrm{T}, \sigma=\mathrm{b}}^{\mathrm{T}+\mathrm{H}}|\mathrm{F}(s)| \mathrm{dt} .
$$

Let $s_{0}=\sigma_{0}+i t_{0} \epsilon R_{2}$. Then (with a positive parameter $X$ bounded below and also above by constant powers of $T$ )

$$
F\left(s_{0}\right)=\frac{1}{2 \pi i} \int_{b d R_{1}} \frac{F(s)}{s-s_{0}} X^{s-s_{0}} \operatorname{Exp}\left(\left(s-s_{0}\right)^{2}\right) d s
$$

(the integral being taken over the boundary of $R_{1}$ in the anti-clockwise direction) and so

$$
\left.\left|F\left(s_{0}\right)\right| \leq \frac{1}{2 \pi} \int_{b d R_{1}} \frac{|F(s)|}{\left|s-s_{0}\right|} X^{\sigma-\sigma_{0}} \mid \operatorname{Exp}\left(s-s_{0}\right)^{2}\right)|| d s \mid
$$

Horizontal portions contribute $O\left(T^{-1}\right)$ because $\left|\operatorname{Exp}\left(\left(s-s_{0}\right)^{2}\right)\right| \ll \operatorname{Exp}\left(-(\log T)^{2}\right)$. Thus

$$
\left|F\left(s_{0}\right)\right| \ll \frac{X^{a-\sigma_{0}}}{2 \pi} \int_{T}^{T+H} \frac{|F(s)| \operatorname{Exp}\left(-\left(t-t_{0}\right)^{2}\right)}{\left|a-\sigma_{0}+i\left(t-t_{0}\right)\right|} d t
$$




$$
+\frac{X^{b-\sigma_{0}}}{2 \pi} \int_{T}^{T+H} \frac{|F(s)| \operatorname{Exp}\left(-\left(t-t_{0}\right)^{2}\right)}{\left|b-\sigma_{0}+i\left(t-t_{0}\right)\right|} d t+O\left(T^{-1}\right) .
$$

Now

$$
\int_{T-\log T}^{T+H-\log T} \frac{\operatorname{Exp}\left(-\left(t-t_{0}\right)^{2}\right)}{\left|a-\sigma_{0}+i\left(t-t_{0}\right)\right|} d t_{0}
$$

can be split up into $\left|t-t_{0}\right| \leq\left|a-\sigma_{0}\right|$ and $\left|t-t_{0}\right| \geq\left|a-\sigma_{0}\right|$. The first portion gives $\ll 1$ and the second portion gives

$$
\begin{aligned}
& \int_{-\infty}^{\infty} \operatorname{Exp}\left(-\left(t-t_{0}\right)^{2}\right)\left|t-t_{0}\right|^{-1} d t_{0} \ll \log \frac{1}{\sigma_{0}-a} \\
& \left|t-t_{0}\right| \geq\left|a-\sigma_{0}\right|
\end{aligned}
$$

Thus

$$
\begin{aligned}
& \int_{t=T}^{T+H} \int_{t_{0}=T-\log T}^{T+H-\log T}|F(s)| \operatorname{Exp}\left(-\left(t-t_{0}\right)^{2}\right)\left|a-\sigma_{0}+i\left(t-t_{0}\right)\right|^{-1} d t_{0} d t \\
& \ll M_{1} \log \frac{1}{\sigma_{0}-a} .
\end{aligned}
$$

Similarly

$$
\begin{aligned}
& \int_{t=T}^{T+H} \int_{t_{0}=T-\log T}^{T+H-\log T}|F(s)| \operatorname{Exp}\left(-\left(t-t_{0}\right)^{2}\right)\left|b-\sigma_{0}+i\left(t-t_{0}\right)\right|^{-1} d t_{0} d t \\
& \ll M_{2} \log \frac{1}{b-\sigma_{0}} .
\end{aligned}
$$

Collecting we have

$$
\int_{t_{0}=T-\log T}^{T+H-\log T}\left|F\left(s_{0}\right)\right| d t_{0} \ll M_{1} X^{a-\sigma_{0}} \log \frac{1}{\sigma_{0}-a}+M_{2} X^{b-\sigma_{0}} \log \frac{1}{b-\sigma_{0}}+T^{-1} .
$$

Here we can choose $X, \delta, \eta$ such that on the horizontal sides of $R_{1}$, the contribution is $O\left(T^{-1)}\right.$ (or some other smaller function of $T$ if necessary). Now

$$
\left.\int_{a+\delta}^{b-\eta} \log \frac{1}{\sigma_{0}-a} d \sigma_{0} \leq \int_{a+\delta}^{b-\eta}\left(\frac{1}{\sigma_{0}-a}\right)^{\frac{1}{2}} d \sigma_{0}=2\left(\sigma_{0}-a\right)^{\frac{1}{2}}\right]_{\sigma_{0}=a+\delta}^{\sigma_{0}=b-\eta}
$$

and a similar bound for $\int_{a+\delta}^{b-\eta} \log \frac{1}{b-\sigma_{0}} d \sigma_{0}$. We remark that if $\left|F^{\prime}(s)\right|$ is not too big then

$$
\int_{T, \sigma=a+\delta}^{T+H}|F(s)| d t
$$


is not very different from the same integral for any $\sigma$ in $a \leq \sigma \leq a+\delta$. Thus

$$
\int_{a}^{a+\delta} \int_{T}^{T+H}|F(s)| d t d \sigma \leq \delta M_{1}
$$

and a similar thing for

$$
\int_{b-\eta}^{b} \int_{T}^{T+H}|F(s)| d t d \sigma \leq \eta M_{2}
$$

For our purposes it suffices to choose $F(s)=\left(\zeta(s, a)-a^{-s}\right)^{2}$, and $X=1$ and this gives Lemma 5.1. It may be noted that by convexity principles we can bound mean square value of derivatives of $\sum_{n=1}^{\infty} \frac{a_{n} b_{n}}{(n+a)^{s}}$. For some more convexity results see $[\mathrm{KR}]_{4}$. 


\section{REFERENCES}

[RB] R.BALASUBRAMANIAN, An improvement of a theorem of Titchmarsh on the mean square of $\left|\zeta\left(\frac{1}{2}+i t\right)\right|$, Proc.London Math.Soc. (3) 36(1978), 540-576.

$[\mathrm{RB}, \mathrm{KR}]_{1}$, R.BALASUBRAMANIAN and K.RAMACHANDRA, Hardy-Littlewood first approximation theorem for quasi L-functions, Hardy-Ramanuajan J., Vol. 27(2004), 1

$[R B, K R]_{2}$ R.BALASUBRAMANIAN and K.RAMACHANDRA, An alternative approach to a theorem of Tom Meurmann, Acta Arith., LV(1990), 351-364.

[EL], EDMUND LANDAU, Handbuch der primzahlen.

[KF] KEVIN FORD, Vinogradov's integral and bounds for the Riemann zeta-function, Proc. London. Math. Soc., (3) 85 (2002), 565-633.

[MNH] M.N.HUXLEY, Exponential sums and the Riemann zeta-function V, Proc. London Math. Soc., (3) 90 (2005), 1-41.

[AI] A.IVIC, Mean Values of the Riemann zeta-function, TIFR (BOMBAY 1991).

[MJ] M.JUTILA (private communication)

$[\mathrm{KR}]_{1}$ K.RAMACHANDRA, Riemann zeta-function, Ramanujan Institute (1979), (A phamplet with 16 pages).

$[\mathrm{KR}]_{2}$ K.RAMACHANDRA, A remark on perron's formula, J.Indian Math.Soc., vol. 65, Nos 1-4(1998) 145-151.

$[\mathrm{KR}]_{3}$ K.RAMACHANDRA, Some remarks on a theorem of Montgomery and Vaughan, J.Number Theory, 11(1979), 465-471. (CHOWLA's anniversary issue).

$[\mathrm{KR}]_{4}$ K.RAMACHANDRA, Mean-value and omega-theorems for the Riemann zeta-function, TIFR Lecture Notes, Springer-Verlag, Berlin-Heidelberg-New York- Tokyo (1995).

$[\mathrm{KR}, \mathrm{AS}]_{1}$ K.RAMACHANDRA and A.SANKARANARAYANAN, A remark on Vinogradov's mean value theorem, the J. of analysis (1995), 111-129.

$[\mathrm{KR}, \mathrm{AS}]_{2}$ K.RAMACHANDRA and A.SANKARANARAYANAN, On an asymptotic formula of Srinivasa Ramanujan, Acta Arith. 109.4 (2003), 349-357. 
[ECT] E.C.TITCHMARSH, The theory of the Riemann zeta-function (second edn. revised and edited by D.R.HEATH-BROWN, Clanendon Press, OXFORD (1986)).

[NZ] N.ZAVOROTNYI, On the fourth power moment of the Riemann zeta-function, (Russian) Preprint, Vladivostok, Far eastern research centre of the Academy of Sciences of USSR(1986), 36 pp.

\section{ADDRESS OF THE AUTHORS}

R. Balasubramanian,

Director,

MATSCIENCE,

Tharamani P.O. 600113,

Chennai, Tamil Nadu

India.

e-mail: balu@imsc.ernet.in

K. Ramachandra

Hon. Vis. Professor

Nat. Inst. of Adv. Studies

I. I. Sc. Campus

Bangalore-560012

Karnataka

India

Also

K. Ramachandra

Retd. Senior Professor

TIFR Centre

P.O.Box 1234

I. I. Sc. Campus

Bangalore-560012

Karnataka

India

e-mail: kram@math.tifrbng.res.in 